\title{
NEITHER FIRST COUNTABLE NOR ČECH-COMPLETE SPACES ARE MAXIMAL TYCHONOFF CONNECTED
}

\author{
D. B. SHAKHMATOV, M. G. TKAČENKO, V. V. TKACHUK, \\ S. WATSON, AND R. G. WILSON
}

(Communicated by Franklin D. Tall)

\begin{abstract}
A connected Tychonoff space $X$ is called maximal Tychonoff connected if there is no strictly finer Tychonoff connected topology on $X$. We show that if $X$ is a connected Tychonoff space and $X \in$ \{locally separable spaces, locally Cech-complete spaces, first countable spaces\}, then $X$ is not maximal Tychonoff connected. This result is new even in the cases where $X$ is compact or metrizable.
\end{abstract}

\section{INTRODUCTION}

A connected space $X$ is called maximal connected if no strictly finer topology on $X$ is connected. The concept was introduced in [15], where $T_{1}$ examples of such spaces were constructed. Later, maximal connected spaces were studied in [7], [8] and [9]. In this last paper and in [14], maximal connected strengthenings of the usual topology on the real line $\mathbb{R}$ were constructed; the space $\mathbb{R}$ being Hausdorff, any maximal connected strengthening is Hausdorff as well. In [13] a characterization of maximal connected $T_{1}$-spaces was given which implies that such spaces must be submaximal (that is, every dense subset is open), an observation which has been made by various authors. However, very few non-trivial examples of submaximal $T_{3}$-spaces are known (see for example [4]), and all known are (at least) totally disconnected. While it is still an open question as to whether there exists a connected submaximal $T_{3}$-space, it is known that any infinite submaximal Tychonoff space which is either first countable, separable or compact is totally disconnected (see [3, $4.8,4.12$ and 5.10], where even stronger results are given).

In view of these results it is natural to ask whether there is a maximal Tychonoff connected space, that is, an infinite connected Tychonoff space $X$ such that any stronger Tychonoff topology on $X$ is disconnected. Such spaces do not apparently have to be submaximal and the results of [3] mentioned in the preceding paragraph do not apply. Even so, the folklore suspicion is that such spaces do not exist. The first steps of an attempted proof were taken in [10], where it was shown that there

Received by the editors April 8, 1996.

1991 Mathematics Subject Classification. Primary 54H11, 54C10, 22A05, 54D06; Secondary $54 \mathrm{D} 25,54 \mathrm{C} 25$.

Key words and phrases. Connected space, separable space, Čech-complete space, first countable space, finer connected topology, maximal connected topology.

The research of the second, third, fourth, and fifth authors was supported by the Mexican National Council of Science and Technology (CONACYT), Grant no. 4874E-9406. 
exists a connected group topology on the reals $\mathbb{R}$ which is stronger than the usual one (see also Example 2.10 of [1]); in [16] stronger connected group topologies were constructed for certain Abelian topological groups.

In this paper we prove that if $X$ is a first countable or a separable or a locally Cech-complete infinite connected Tychonoff space, then it has a strictly finer connected Tychonoff topology, that is, it is not maximal Tychonoff connected. The results are new even for the classes of metrizable or compact spaces.

A well-known class containing all first countable and locally Čech-complete spaces is the class of spaces of pointwise countable type. While we did not succeed in proving that all spaces with this property are not maximal Tychonoff connected, we do establish this result when, additionally, the space has countable cellularity.

Throughout, $I$ will denote the unit interval $[0,1]$ with the usual metric topology; all spaces are Tychonoff. Where not defined, cardinal function notation is taken from [11]. Recall that a subset $U$ of a space $X$ is said to be regular open in $X$ if $X \backslash U=c l_{X}\left(X \backslash c l_{X} U\right)$ or equivalently, $U=\operatorname{Int}_{X}\left(c l_{X} U\right)$.

\section{The RESUlts}

The following technical lemma, which is fundamental to the main theorems, is a modification of Proposition 4.2 of [16].

Lemma 1. Let $X, Y$ be connected spaces and $S$ a dense subset of the product $\Pi=X \times Y$ with $\pi_{X}(S)=X$, where $\pi_{X}: X \times Y \rightarrow X$ is the projection. If $U$ and $V$ are non-empty disjoint open sets in $X \times Y$ with $S \subseteq U \cup V$ and $\Phi=c_{\Pi} U \cap c l_{\Pi} V$, then the set $\pi_{X}(\Phi)$ has a non-empty interior in $X$. Further, if both $U$ and $V$ are regular open in $X \times Y$ then there exists a non-empty open set $W$ in $X$ such that $W \subseteq \pi_{X}(\Phi)$ and $U \cap \pi_{X}^{-1}(x) \neq \emptyset \neq V \cap \pi_{X}^{-1}(x)$ for all $x \in W$.

Proof. Note first that the sets $S$ and $\Phi$ are disjoint because $S$ is covered by $U$ and $V$. Let $x \in X \backslash \pi_{X}(\Phi)$; then $\pi_{X}^{-1}(x) \cap \Phi=\emptyset$ and we claim that either $\pi_{X}^{-1}(x) \subseteq c l_{\Pi} U$ or $\pi_{X}^{-1}(x) \subseteq c l_{\Pi} V$ : For otherwise, the equality

$$
\pi_{X}^{-1}(x)=\left(\pi_{X}^{-1}(x) \cap c l_{\Pi} U\right) \cup\left(\pi_{X}^{-1}(x) \cap c l_{\Pi} V\right)
$$

would give us a partition of $\pi_{X}^{-1}(x)$ into two disjoint clopen subsets, which contradicts the connectedness of the space $\pi_{X}^{-1}(x) \cong Y$.

For a subset $O \subseteq X \times Y$, define

$$
\pi_{X}^{\sharp}(O)=X \backslash \pi_{X}(X \times Y \backslash O) .
$$

It is clear that $\pi_{X}^{\sharp}(O)=\left\{x \in X: \pi_{X}^{-1}(x) \subseteq O\right\}$. We claim that:

$$
X \backslash \pi_{X}(\Phi) \subseteq \pi_{X}^{\sharp}\left(c l_{\Pi} U\right) \cup \pi_{X}^{\sharp}\left(c l_{\Pi} V\right) .
$$

To prove this, let $x \in X \backslash \pi_{X}(\Phi)$. Then either $\pi_{X}^{-1}(x) \subseteq c l_{\Pi} U$ or $\pi_{X}^{-1}(x) \subseteq c l_{\Pi} V$, whence either $x \in \pi_{X}^{\sharp}\left(c l_{\Pi} U\right)$ or $x \in \pi_{X}^{\sharp}\left(c l_{\Pi} V\right)$, which proves (1).

Since the projection $\pi_{X}$ is open, both sets $\pi_{X}^{\sharp}\left(c l_{\Pi} U\right)$ and $\pi_{X}^{\sharp}\left(c l_{\Pi} V\right)$ are closed in $X$. We claim that they are disjoint. Indeed, if $x \in \pi_{X}^{\sharp}\left(c l_{\Pi} U\right) \cap \pi_{X}^{\sharp}\left(c l_{\Pi} V\right)$, then $\pi_{X}^{-1}(x) \subseteq c l_{\Pi} U \cap c l_{\Pi} V=\Phi$. Since $\pi_{X}(S)=X$, there exists $y \in Y$ with $(x, y) \in S$; but then $(x, y) \in S \cap \Phi \neq \emptyset$, a contradiction.

Note that $\pi_{X}^{\sharp}\left(c l_{\Pi} U\right) \neq X \neq \pi_{X}^{\sharp}\left(c l_{\Pi} V\right)$; otherwise either $c l_{\Pi} U=X \times Y$ or $c_{\Pi} V=X \times Y$ which is impossible, because $U$ and $V$ are non-empty. Since $X$ is 
connected, we conclude that

$$
X \neq \pi_{X}^{\sharp}\left(c l_{\Pi} U\right) \cup \pi_{X}^{\sharp}\left(c l_{\Pi} V\right) .
$$

Therefore, (1) implies that the non-empty open set $W=X \backslash\left(\pi_{X}^{\sharp}\left(c l_{\Pi} U\right) \cup \pi_{X}^{\sharp}\left(c l_{\Pi} V\right)\right)$ is contained in $\pi_{X}(\Phi)$. This proves the first part of the lemma.

Suppose that both $U$ and $V$ are regular open sets in $X \times Y$ and pick an arbitrary point $x$ in the set $W$ just defined. From the definition of $W$ it follows that $\pi_{X}^{-1}(x) \backslash$ $c l_{\Pi} U \neq \emptyset$ and $\pi_{X}^{-1}(x) \backslash c l_{\Pi} V \neq \emptyset$. The sets $U$ and $V$ being regular open, we have $U=(X \times Y) \backslash c l_{\Pi} V$ and $V=(X \times Y) \backslash c l_{\Pi} U$. Therefore, $\pi_{X}^{-1}(x) \cap U \neq \emptyset$ and $\pi_{X}^{-1}(x) \cap V \neq \emptyset$.

The following result is well-known in folklore, but we give its proof here for the sake of completeness.

Lemma 2. Let $O$ be a non-empty open subset of a Čech-complete space $X$ without isolated points. Then there exists a non-empty compact separable subset $F$ of $X$ which has no isolated points and is contained in $O$.

Proof. Let $\beta X$ be the Čech-Stone compactification of $X$. Since $X$ is Čech-complete, $X$ is a $G_{\delta}$-set in $\beta X$, so that there exists a decreasing sequence $\left\{U_{n}: n \in \omega\right\}$ of open sets in $\beta X$ such that $X=\bigcap_{n \in \omega} U_{n}$.

Denote by $\mathcal{F}=\bigcup_{n \in \omega}\{0,1\}^{n}$ the Cantor tree. For a function $f: n \rightarrow\{0,1\}$ let $f \frown 0$ and $f \frown 1$ be functions from $n+1$ to $\{0,1\}$ that extend $f$ and are equal to 0 and 1 at the point $n$ respectively. Choose an open set $W$ in $\beta X$ with $O=W \cap X$ and find another non-empty open set $O(0)$ in $\beta X$ with $c_{\beta X} O(0) \subseteq W \cap U_{0}$. Suppose that for some $n \in \omega$ we have defined a disjoint family $\left\{O(f): f \in\{0,1\}^{n}\right\}$ of non-empty open subsets of $\beta X$ so that $O(f) \subseteq U_{n}$ for each $f$. Both $X$ and $\beta X$ have no isolated points, so the sets $O(f)$ have no isolated points either. For every $f \in\{0,1\}^{n}$ choose non-empty open subsets $O(f \frown 0)$ and $O(f \frown 1)$ of $\beta X$ so that $c l_{\beta X} O(f \frown 0) \cap c_{\beta X} O(f \frown 1)=\emptyset$ and $c_{\beta X} O(f \frown 0) \cup c l_{\beta X} O(f \frown 1) \subseteq O(f) \cap U_{n+1}$.

Define $F_{n}=\bigcup\left\{c l_{\beta X} O(f): f \in\{0,1\}^{n}\right\}$ for $n \in \omega$, and $F^{*}=\bigcap_{n \in \omega} F_{n}$. Then $F^{*}$ is a closed subset of $\beta X$, and hence is compact. From the choice of the sequence $\left\{U_{n}: n \in \omega\right\}$ and the fact that $c l_{\beta X} O(f) \subseteq U_{n}$ for all $f \in\{0,1\}^{n}, n \in \omega$, it follows that $F^{*} \subseteq X$. Therefore, $F^{*} \subseteq X \cap O(0) \subseteq X \cap W=O$.

The construction of the set $F^{*}$ enables us to define in a standard way a continuous function $\varphi$ from $F^{*}$ onto the Cantor set $C=\{0,1\}^{\omega}$. Indeed, for a point $x \in F^{*}$ let $\varphi(x)$ be the (unique) function $g \in\{0,1\}^{\omega}$ such that $x \in c_{\beta X} O(g \mid n)$ for each $n \in \omega$. It remains to take an irreducible preimage of $\varphi$, that is, a closed subset $F$ of $F^{*}$ such that $\varphi(F)=C$ and the restriction $\left.\varphi\right|_{F}$ is irreducible. One easily verifies that $F$ is a separable compact space with no isolated points and $F \subseteq F^{*} \subseteq O$.

Lemma 3. If $X$ is a non-empty space with countable cellularity and $w(X) \leq \mathfrak{c}$, then the product space $X \times I$ has precisely $\mathfrak{c}$ regular open sets.

Proof. That $X \times I$ has at least $\mathfrak{c}$ regular open sets follows from the fact that $I$ has $\mathfrak{c}$ regular open sets.

Let $\mathcal{B}$ be a base of power $\kappa$ in $X$, where $\kappa \leq \mathfrak{c}$. Say that an open subset $U$ of the product $X \times I$ is $\mathcal{B}$-generated if $U=\bigcup\left\{U_{n}: n \in \omega\right\}$, where each $U_{n}$ has the form $V_{n} \times W_{n}$ with $V_{n} \in \mathcal{B}$ and $W_{n}$ a rational interval of $I$.

There are $\kappa^{\omega} \leq \mathfrak{c}$ countable subfamilies of $\mathcal{B}$ and consequently at most $\mathfrak{c}$ many $\mathcal{B}$-generated open subsets of $X \times I$. Since $I$ is second countable, it follows that 
$c(X \times I)=\aleph_{0}$. Now let $W$ be a regular open subset of $X \times I$ and let $\mathcal{F}_{W}$ be a maximal (necessarily countable) disjoint family of $\mathcal{B}$-generated open sets which are contained in $W$. Clearly $\bigcup \mathcal{F}_{W}$ is dense in $W$ and hence $W=\operatorname{Int}\left(\operatorname{cl}\left(\bigcup \mathcal{F}_{W}\right)\right)$. Thus each regular open set in $X \times I$ is determined by a countable subset of $\mathcal{B}$ and we are done.

Corollary 1. If a non-empty space $X$ has countable cellularity and character, then the product $X \times I$ has precisely $\mathfrak{c}$ regular open sets.

Proof. By [11, 2.16], $|X| \leq 2^{c(X) \chi(X)}=2^{\aleph_{0}}$. For each $x \in X$, let $\mathcal{B}_{x}$ be a countable local base at $x$ and let $\mathcal{B}=\bigcup \mathcal{B}_{x}$. Then $\mathcal{B}$ is a base for $X$ of cardinality no greater than $\mathfrak{c}$; the result now follows from Lemma 3 .

For a given space $X$, denote by $\triangle(X)$ the least power of non-empty open sets in $X$. One of the main results of the paper is the following theorem.

Theorem 1. Let $X$ be a connected Tychonoff space and suppose that $X$ has a family $\mathcal{F}=\left\{F_{i}: i \in A\right\}$ of subsets such that

(1) $\triangle\left(F_{i}\right) \geq \mathfrak{c}$ for each $i \in A$,

(2) $w\left(F_{i}\right) \leq \mathfrak{c}$ for each $i \in A$,

(3) $c\left(F_{i}\right)=\aleph_{0}$ for each $i \in A$ and

(4) $\bigcup \mathcal{F}$ is dense in $X$.

Then there is a dense connected subspace $Y \subseteq X \times I$ such that $\pi_{X}(Y)=X$ and $\left|\pi_{X}^{-1}(x) \cap Y\right|=1$ for every $x \in X$ (that is, the projection $\pi_{X}$ is a bijection between $Y$ and $X)$. In particular, $X$ admits a strictly finer connected Tychonoff topology.

Proof. Let $\left\{F_{\delta}: \delta<\lambda\right\}$ be a well-ordering of $\mathcal{F}$ and define inductively a disjoint family $\left\{G_{\delta}: \delta<\lambda\right\}$ as follows:

Let $G_{0}=F_{0}$ and for some $\xi<\lambda$, suppose that we have defined $G_{\delta}$ for each $\delta<\xi$; let

$$
G_{\xi}=F_{\xi} \backslash c l\left(\bigcup_{\delta<\xi} G_{\delta}\right) .
$$

For each $\delta<\lambda, G_{\delta}$ is an open (possibly empty) subset of $F_{\delta}$ and hence the family $\mathcal{G}=\left\{G_{\delta}: \delta<\lambda\right\}$ is disjoint and satisfies the conditions (1), (2) and (3) of the theorem. Furthermore, $\operatorname{cl}\left(\bigcup_{\delta<\lambda} G_{\delta}\right)=\operatorname{cl}\left(\bigcup_{\delta<\lambda} F_{\delta}\right)$, and hence (4) is satisfied as well. In what follows, we assume that the family $\mathcal{G}$ is indexed by $A$.

For every $i \in A$ we will define a (discontinuous) function $f_{i}: G_{i} \rightarrow I$. We use Lemma 3 to enumerate all pairs $(U, V)$ of non-empty regular open sets in the product $G_{i} \times I$, say $\left\{\left(U_{\alpha}^{i}, V_{\alpha}^{i}\right): \alpha<\mathfrak{c}\right\}$. Let $\beta<\mathfrak{c}$ be an ordinal and suppose that we have defined points $x_{\alpha} \in G_{i}$ and the values $f_{i}\left(x_{\alpha}\right)$ of $f_{i}$ at these points for each $\alpha<\beta$. Denote by $\pi_{X}$ the projection of $X \times I$ onto $X$. If the cardinality of the set $P_{\beta}=\pi_{X}\left(\left(G_{i} \times I\right) \backslash\left(U_{\beta}^{i} \cup V_{\beta}^{i}\right)\right)$ is at least $\mathfrak{c}$, choose points $x_{\beta} \in G_{i} \backslash\left\{x_{\alpha}: \alpha<\beta\right\}$ and $y_{\beta} \in I$ so that $\left(x_{\beta}, y_{\beta}\right) \notin\left(U_{\beta}^{i} \cup V_{\beta}^{i}\right)$ and put $f_{i}\left(x_{\beta}\right)=y_{\beta}$. Otherwise pick an arbitrary point $x_{\beta} \in G_{i} \backslash\left\{x_{\alpha}: \alpha<\beta\right\}$ and put $f_{i}\left(x_{\beta}\right)=0$.

If $H_{i}=G_{i} \backslash\left\{x_{\alpha}: \alpha<\mathfrak{c}\right\} \neq \emptyset$, extend $f_{i}$ to $G_{i}$ by defining $f_{i}(x)=0$ for each $x \in H_{i}$. The graph of $f_{i}$ is dense in $G_{i} \times I$, and hence $f_{i}$ is discontinuous. Indeed, let $O$ be a non-empty open subset of $G_{i} \times I$. By the regularity of $G_{i}$ and $I$ there exist non-empty regular open sets $O_{1} \subseteq G_{i}$ and $O_{2} \subseteq I$ such that the closure of $O_{1} \times O_{2}$ is a proper subset of $O$. The latter is possible, for $G_{i} \times I$ has no isolated points. 
Both sets $O^{\prime}=O_{1} \times O_{2}$ and $O^{\prime \prime}=\left(G_{i} \times I\right) \backslash c l O^{\prime}$ are non-empty and regular open in $G_{i} \times I$, so that $\left(O^{\prime}, O^{\prime \prime}\right)=\left(U_{\alpha}^{i}, V_{\alpha}^{i}\right)$ for some $\alpha<\gamma$. The set $\pi_{X}\left(\left(G_{i} \times I\right) \backslash\left(U_{\alpha}^{i} \cup V_{\alpha}^{i}\right)\right)$ has at least $\mathfrak{c}$ points and therefore, $\left(x_{\alpha}, f\left(x_{\alpha}\right)\right) \in\left(G_{i} \times I\right) \backslash O^{\prime \prime}=c l O^{\prime} \subseteq O$. This proves the density of the graph of $f_{i}$ in $G_{i} \times I$.

Since the elements of $\mathcal{G}$ are disjoint, we can define $f: X \rightarrow I$ by $\left.f\right|_{G_{i}}=f_{i}$ for $i \in A$ and $f(x)=0$ for each $x \in X \backslash \bigcup \mathcal{G}$. Consider the subspace $G r(f)=$ $\{(x, f(x)): x \in X\}$ of $X \times I$. The restriction $p$ of the projection $\pi_{X}: X \times I \rightarrow X$ to $G r(f)$ is a continuous one-to-one mapping of $G r(f)$ onto $X$. Since $f$ extends each function $f_{i}$ and the graph of $f_{i}$ is dense in $G_{i} \times I$ for each $i \in A$, the graph of $f$, $G r(f)$, is dense in $X \times I$. Therefore, $p$ is not a homeomorphism between $\operatorname{Gr}(f)$ and $X$. We claim that the space $G r(f)$ is connected. Assume the contrary, and let $U$ and $V$ be non-empty disjoint open subsets of $X \times I$ which cover $G r(f)$. The interiors of the closures of these sets (in $X \times I$ ) are also non-empty disjoint and open, so we can assume that both $U$ and $V$ are regular open. Denote $\Phi=c l U \cap c l V$. Choose an open non-empty set $W \subseteq \pi_{X}(\Phi)$ as in Lemma 1. The space $X$ being connected, all non-empty open subsets of $X$ are of cardinality at least $\mathfrak{c}$, that is, $\triangle(X) \geq \mathfrak{c}$. Since $\bigcup \mathcal{G}$ is dense in $X$, there is an $i \in A$ such that $W \cap G_{i} \neq \emptyset$. Therefore, $\emptyset \neq U \cap\left(G_{i} \times I\right) \subseteq U_{\alpha}^{i}$ and $\emptyset \neq V \cap\left(G_{i} \times I\right) \subseteq V_{\alpha}^{i}$ for some $\alpha<\gamma$ and at the $\alpha$-th step of the recursive construction we have $\left(x_{\alpha}, f\left(x_{\alpha}\right)\right)=\left(x_{\alpha}, f_{i}\left(x_{\alpha}\right)\right) \in G r\left(f_{i}\right) \backslash(U \cup V)$. This contradicts the fact that the $\left(x_{\alpha}, f\left(x_{\alpha}\right)\right) \in G r\left(f_{i}\right) \subseteq G r(f) \subseteq U \cup V$. Now let $Y=G r(f)$. It is clear that $Y$ is as required and this completes the proof.

Note that if each $F_{i}$ in the above theorem is separable, then conditions 2) and 3) of the theorem are satisfied. Hence combining Theorem 1 and Lemma 2, we obtain the following result.

Theorem 2. Every infinite connected locally Čech-complete space admits a strictly finer connected Tychonoff topology.

Theorem 3. If an infinite connected Tychonoff space $X$ contains a dense Čechcomplete subspace, then $X$ admits a strictly finer connected Tychonoff topology.

Corollary 2. Every infinite regular connected locally compact space admits a strictly finer connected Tychonoff topology.

Corollary 3. Every infinite locally separable connected Tychonoff space admits a strictly finer locally separable connected Tychonoff topology.

Recall that a space $X$ has pointwise countable type if it is the union of compact subspaces each having a countable neighbourhood base in $X$.

Theorem 4. Let $X$ be an infinite connected Tychonoff space of pointwise countable type and countable cellularity. Then $X$ has a strictly finer connected Tychonoff topology.

Proof. Let $\Gamma$ be the family of all compact dense-in-themselves subsets of $X$. Denote by $F=\operatorname{cl}(\bigcup \Gamma)$ and let $U=X \backslash F$. It is clear that:

(1) By Lemma 2, the space $F$ admits a family $\mathcal{F}$ satisfying conditions (1) through (4) of Theorem 1 , and

(2) If $U=\emptyset$ then we can apply Theorem 1 and we are done. Otherwise, if $U \neq \emptyset$, then every compact subset of $U$ is scattered.

If $U \neq \emptyset$, then denote by $M$ the set of points of $U$ which have countable character. 
Claim (i). The set $M$ is $G_{\delta}$-dense in $U$; that is, each non-empty $G_{\delta}$-set in $U$ meets $M$. To prove this, suppose that $H$ is a $G_{\delta}$ in $U$ and let $x \in H$. Then $x$ is contained in a compact subspace $P$ of $X$ of countable character in $X$. There exists a compact $G_{\delta}$-subset $H_{1}$ of $X$ with $x \in H_{1} \subseteq H \cap P$. Clearly, $H_{1}$ is of countable character in $P$ and since the compact set $P$ is of countable character in $X$, it follows that $H_{1}$ has countable character in $X$ (see 3.1.E of [5]). Now by (2) above, it follows that $H_{1}$ is scattered and hence has an isolated point $y$. Then $y \in M \cap H$ and our first claim is proved.

Claim (ii). Every non-empty open subset of $M$ has cardinality c. To prove this, let $W$ be a non-empty open subset of $M$; then there is an open $V$ in $U$ with $V \cap M=W$. Take any point $x \in W$ and choose a continuous function $f: X \rightarrow I$ with $f(x)=1$ and $f(X \backslash V)=\{0\}$.

The space $X$ is connected, so that $f(X)=I$. To show that $W$ has cardinality at least $\mathfrak{c}$, it clearly suffices to establish that $f(W) \supseteq(0,1)$. To this end, pick any $r \in(0,1)$; the set $H=f^{-1}(r)$ is a non-empty $G_{\delta}$ in $X$ (and hence in $U$ ) and is contained in $V$ and hence in $U$. It follows immediately from Claim $(i)$ that $H$ meets $W$. Finally, we note that since $c(M)=\chi(M)=\aleph_{0}$, it follows from $[11,2.16]$ that $|M|=\mathfrak{c}$ and our second claim is proved. Note also that $w(M) \leq|M| \cdot \chi(M) \leq \mathfrak{c}$.

Finally, from Corollary 1 it follows that $M \times I$ has precisely $\mathfrak{c}$ regular open sets. Now let $\mathcal{G}=\mathcal{F} \cup\{M\} ; \mathcal{G}$ satisfies the conditions (1) through (4) of Theorem 1 and we are done.

Problem 1. Does every infinite connected Čech-complete space admit a strictly finer connected Čech-complete topology?

Problem 2. Does every infinite connected Tychonoff space of point-countable type admit a strictly finer connected topology?

Problem 3. If $X$ is an infinite connected countably compact (pseudocompact) Tychonoff space, is there a strictly finer connected Tychonoff topology on $X$ ?

Problem 4. Does every infinite compact connected space admit a strictly finer connected Lindelöf (normal) topology?

The following result, often referred to as Kuratowski's theorem, is part of the folklore; a proof may be found in [12, Section 1.5].

Lemma 4. Let $X$ be a connected topological space and $C \subseteq X$ a connected subspace. If $S$ is a component of $X \backslash C$, then $X \backslash S$ is connected.

Corollary 4. Every infinite connected topological space $X$ is the union of two nonempty disjoint connected subspaces.

Proof. With the same notation as in the lemma, $X$ is the union of $S$ and $X \backslash S$ (one can take $C$ to be a singleton).

Definition. Say that a Tychonoff space $X$ is unruly at $x \in X$ if $X \backslash\{x\}$ is not $C^{*}$-embedded in $X$. Say that $X$ is unruly if it is unruly at each non-isolated point, and $X$ is hereditarily unruly if each subspace of $X$ is unruly.

It is well known that for any Tychonoff space $X$, no point of $\beta X \backslash X$ is a point of first countability. We now state this theorem in a slightly different form and, for completeness, give its proof. 
Lemma 5. Every first countable Tychonoff space is hereditarily unruly.

Proof. Let $X$ be a first countable Tychonoff space; since the property of being first countable is hereditary, it clearly suffices to show that $X$ is unruly. To this end, let $x$ be a non-isolated point in $X$. Choose a nested local base $\mathcal{B}_{x}=\left\{B_{n}: n \in \omega\right\}$ at $x$. We can assume without loss of generality that for each $n \in \omega$, we have $\operatorname{cl}\left(B_{n+1}\right) \subseteq B_{n}$. Let $A_{n}=B_{n} \backslash \operatorname{cl}\left(B_{n+1}\right)$; clearly $A_{n}$ is non-empty for infinitely many (and we assume for all) $n$ and hence we can choose $x_{n} \in A_{2 n}$ for each $n \in \omega$. Now since $\left\{A_{2 n}: n \in \omega\right\}$ is a discrete family of open subsets of $X \backslash\{x\}$ it follows from [6, Lemma 1.1] that $S=\left\{x_{n}: n \in \omega\right\}$ is $C$-embedded in $X \backslash\{x\}$.

Now define $f: S \rightarrow[0,1]$ by $f\left(x_{n}\right)=0$ if $n$ is odd and $f\left(x_{n}\right)=1$ if $n$ is even.

Let $g$ be a continuous bounded extension of $f$ to $X \backslash\{x\}$. Since $\left\{x_{n}\right\}$ converges to $x$ in $X$, it follows immediately that $g$ has no continuous extension to $X$.

Remark 1. There exist countably compact Fréchet-Urysohn spaces without isolated points which are not unruly at any point: an uncountable $\Sigma$-product of unit intervals is such a space.

Theorem 5. An infinite hereditarily unruly connected Tychonoff space $(X, \tau)$ admits a strictly finer connected Tychonoff topology.

Proof. By Corollary $4, X$ is the union of two disjoint connected non-empty sets, $C$ and $D$ say. Since $(X, \tau)$ is connected, one of these two sets, $D$ say, is not closed and we can choose $c \in \operatorname{cl}(D) \cap C$. Since $X$ is hereditarily unruly, $D$ is not $C^{*}$-embedded in $D \cup\{c\}$. Let $h$ be a bounded continuous function on $D$ which has no continuous extension to $D \cup\{c\}$; then there exists a net $\left\{x_{\alpha}: \alpha \in I\right\}$ in $D$ convergent to $c$ such that $\left\{h\left(x_{\alpha}\right): \alpha \in I\right\}$ is not convergent in $\mathbb{R}$. Then clearly $\left\{x_{\alpha}: \alpha \in I\right\}$ is not convergent in $\beta D$ and hence we can find two subnets of $\left\{x_{\alpha}: \alpha \in I\right\}$ convergent to distinct points $s, t \in \beta D$. Let $g$ be any continuous function on $\beta D$ which separates $s$ and $t$ and define $f: X \rightarrow \mathbb{R}$ by

$$
\begin{gathered}
f(x)=g(s) \text { if } x \in C \text { and } \\
f(x)=g(x) \text { if } x \in D .
\end{gathered}
$$

Clearly $f$ is a discontinuous function on $X$ but $\left.f\right|_{C}$ and $\left.f\right|_{D}$ are both continuous. We denote by $\sigma$ the weak topology on $X$ induced by $C(X) \cup\{f\}$, where $C(X)$ is the family of all continuous functions on $X$. It is clear that $\sigma$ is strictly finer than $\tau$. The space $(X, \sigma)$ is Tychonoff and both $\left(C,\left.\sigma\right|_{C}\right)$ and $\left(D,\left.\sigma\right|_{D}\right)$ are connected, for $\left.\sigma\right|_{C}=\left.\tau\right|_{C}$ and $\left.\sigma\right|_{D}=\left.\tau\right|_{D}$. That $(X, \sigma)$ is connected follows from the fact that every cozero set in $(X, \sigma)$ which contains $c$ meets the net $\left\{x_{\alpha}: \alpha \in I\right\}$ and hence $c \in C \cap \operatorname{cl}_{\sigma}(D)$.

Remark 2. It is worth noting that if the space $(X, \tau)$ in Theorem 5 is first countable or metrizable then so is $(X, \sigma)$. Thus we obtain the following corollaries:

Corollary 5. A first countable infinite connected Tychonoff space admits a strictly finer connected first countable Tychonoff topology, and hence no first countable Tychonoff space is maximal Tychonoff connected.

Alternatively, Corollary 5 can be stated in the following form:

Corollary 6. No infinite Tychonoff space is maximal with respect to being connected and first countable. 
Corollary 7. An infinite (separable) metrizable connected space admits a strictly finer connected (separable) metrizable topology.

Analogous to Corollary 6 we have:

Corollary 8. No infinite space is maximal with respect to being connected and metrizable.

Finally, we study those connected spaces which can be densely embedded in certain Cartesian products. We prove the following:

Theorem 6. Let $X$ be a connected subspace of a product $\Pi=\prod_{\alpha \in A} X_{\alpha}$ of secondcountable Tychonoff spaces which fills all countable faces of $\Pi$. If $|X|>1$ then $X$ admits a strictly finer connected Tychonoff topology.

Proof. For every $\alpha \in A$, let $\pi_{\alpha}: \Pi \rightarrow X_{\alpha}$ be the projection. Pick an index $\alpha_{0} \in A$ such that $\left|X_{\alpha_{0}}\right|>1$. Obviously $X_{\alpha_{0}}$ is infinite and so by Corollary $7, X_{\alpha_{0}}$ admits a strictly finer second-countable connected Tychonoff topology $\sigma_{0}$. Denote $\Pi^{*}=\prod_{\alpha \in A} Y_{\alpha}$, where $Y_{\alpha}=X_{\alpha}$ if $\alpha \in A \backslash\left\{\alpha_{0}\right\}$ and $Y_{\alpha_{0}}=\left(X_{\alpha_{0}}, \sigma_{0}\right)$. Then the product topology $\sigma$ of $\Pi^{*}$ is strictly finer than the product topology $\tau$ of $\Pi$. Further, the restriction $\left.\sigma\right|_{X}$ of $\sigma$ to $X$ is strictly finer than $\left.\tau\right|_{X}$. Indeed, by hypothesis $X$ fills all countable faces of $\Pi$, and hence the restriction $\left.\pi_{\alpha}\right|_{X}$ of $\pi_{\alpha}$ to $X$ is open for each $\alpha \in A$. In particular, the map $\left.\pi_{\alpha_{0}}\right|_{X}$ of $X$ onto $X_{\alpha_{0}}$ is open. If the topologies $\left.\sigma\right|_{X}$ and $\left.\tau\right|_{X}$ were the same, the set $\pi_{\alpha_{0}}\left(X \cap \pi_{\alpha_{0}}^{-1} U\right)=U$ would be open in $X_{\alpha_{0}}$ for every $\sigma_{\alpha_{0}}$-open set $U$ in $X_{\alpha_{0}}$; that is, $\sigma_{\alpha_{0}}$ would coincide with the original topology of $X_{\alpha_{0}}$.

It remains to show that $\left(X,\left.\sigma\right|_{X}\right)$ is connected. If not, there exists a continuous function $f$ of $\left(X,\left.\sigma\right|_{X}\right)$ onto the discrete space $\{0,1\}$. Since $X$ fills all countable faces in $\Pi^{*}$, it is dense in $\Pi^{*}$. Furthermore, all factors of the product $\Pi^{*}$ are second-countable, so by Theorem 1 of [2] the function $f$ depends on countably many coordinates. That is to say, there exists a countable subset $B \subseteq A$ such that for all $x, y \in X$ the equality $\pi_{B}(x)=\pi_{B}(y)$ implies $f(x)=f(y)$ (here $\pi_{B}$ is the projection of $\Pi^{*}$ onto $\left.\prod_{\alpha \in B} Y_{\alpha}\right)$. This makes it possible to define a function $g: \pi_{B}(X) \rightarrow\{0,1\}$ such that $f=\left.g \circ \pi_{B}\right|_{X}$. The restriction $\left.\pi_{B}\right|_{X}: X \rightarrow \prod_{\alpha \in B} Y_{\alpha}$ is open ( $X$ is endowed with the topology $\left.\sigma\right|_{X}$ ), and hence $g$ is also continuous. Note that $\pi_{B}(X)=\prod_{\alpha \in B} Y_{\alpha}$ is connected being a product of connected spaces, but the image $g\left(\pi_{B}(X)\right)=\{0,1\}$ is not. This contradiction completes the proof.

Problem 5. Does Theorem 6 remain valid if one assumes only that $X$ fills all finite faces of $\Pi$ ?

\section{REFERENCES}

[1] Alas, O. T., Tkačenko, M. G., Tkachuk, V. V., and Wilson R. G., Connectedness and local connectedness of topological groups and extensions, submitted, 1995.

[2] Arhangel'skiı̌, A. V., On mappings of everywhere dense subspaces of topological products, Dokl. Acad. Nauk SSSR 197 (1971), 750-756. MR 43:6866

[3] Arhangel'skǐ̌, A. V. and Collins, P., On submaximal spaces, Topology Appl., 64 (1995), 219-242. MR 96m:54002

[4] van Douwen, E. K., Applications of maximal topologies, Topology Appl. 51 (1993), 125-139. MR 94h:54012

[5] Engelking, R., General Topology. PWN, Warszawa 1977. MR 58:18316b

[6] García-Máynez, A. and Wilson, R. G., Classes of topological spaces preserved under realcompactifications, Bol. Soc. Mat. Mex., vol. 31 (1986), 57-60. MR 89k:54057 
[7] Guthrie, J. A., Reynolds, D. F. and Stone, H. E., Connected expansions of topologies, Bull. Austral. Math. Soc. 9 (1973), 259-265. MR 48:7201

[8] Guthrie, J. A. and Stone, H. E., Spaces whose connected expansions preserve connected subsets, Fund. Math. 80 (1973), 91-100. MR 48:9653

[9] Guthrie, J. A., Stone, H. E. and Wage, M.L., Maximal connected expansions of the reals, Proc. Amer. Math. Soc. 69 (1978), 159-165. MR 57:7501

[10] Jones, F. B., Connected and disconnected sets and the functional equation $f(x)+f(y)=$ $f(x+y)$, Proc. Amer. Math. Soc. 48 (1942), 115-120.

[11] Juhász, I., Cardinal Functions in Topology. Math. Centrum Tracts, no. 34, Amsterdam, 1971. MR 49:4778

[12] Kok, H., Connected Orderable Spaces. Math. Centrum Tracts, no. 49, Amsterdam, 1973. MR 49:3862

[13] Neumann-Lara, V. and Wilson, R. G., Some properties of essentially connected and maximally connected spaces, Houston J. Math. 12 (1986), 419-429. MR 88a:54049

[14] Simon, P., An example of maximal connected Hausdorff space, Fund. Math. 100 (1978), 157-163. MR 58:7557

[15] Thomas, J. P., Maximal connected topologies, J. Austral. Math. Soc. 8 (1968), 700-705. MR 38:5177

[16] Tkačenko, M. G. and Villegas-Silva, L. M., Refining connected topological group topologies on Abelian torsion-free groups, submitted, 1995.

(D. B. Shakhmatov) Department of Mathematics, Faculty of Science, Ehime UniverSity, MatsuYAma 790, JAPAN

E-mail address: dmitri@dpc.ehime-u.ac.jp

(M. G. Tkačenko, V. V. Tkachuk, and R. G. Wilson) Departamento de Matemáticas, Universidad Autónoma Metropolitana, Av. Michoacán y la Purísima, Iztapalapa, A.P. 55532, C.P. 09340, MÉXico, D.F.

E-mail address, M. G. Tkačenko: mich@xanum.uam.mx

E-mail address, V. V. Tkachuk: vova@xanum.uam.mx

E-mail address, R. G. Wilson: rgw@xanum.uam.mx

(S. Watson) Department of Mathematics, York University, North York, Ontario, CANADA M3J 1P3

E-mail address: stephen.watson@mathstat.yorku.ca 